\section{Extremophilic actinomycetes isolated from soil in Kazakhstan: Classification and antimicrobial activities}

\author{
Ainur Zhulamanova, 1,2 \\ Zhanat Koshenov, 1,2,3 \\ Saikal Shamkeeva, 1,4 \\ Zhannur Markhametova, ${ }^{1}$ \\ Diyora Abdukhakimova, ${ }^{1}$ \\ Lyudmila Trenozhnikova, 5 \\ Azliyati Azizan ${ }^{1}$
}

${ }^{1}$ Nazarbayev University School of Medicine (NUSOM), Nur-Sultan, Kazakhstan; ${ }^{2}$ National Laboratory Astana, (NLA), Nazarbayev University, Nur-Sultan, Kazakhstan; ${ }^{3}$ Medical University of Graz, Gottfried Schatz

Research Center, Department of Molecular Biology and Biochemistry, Graz, Austria; ${ }^{4}$ Leipzig University, Institute for Laboratory Medicine, Clinical Chemistry and Molecular Diagnostics, Lepzig, Germany; ${ }^{5}$ Research and Production Center for Microbiology and Virology (RPCMV), Almaty, Kazakhstan

\begin{abstract}
Extremophilic actinomycetes species are capable of surviving in extreme environment and producing antibiotics. In this study, we hypothesize that extremophiles produce antimicrobial compounds that are potentially novel agent(s) effective against drug resistant pathogens. The goal of this study is to test inhibitory activity of the extracts derived from extremophilic actinomycetes species against the most prevalent drug-resistant bacteria in Kazakhstani hospitals, and preliminarily analyze chemical composition of the active extracts. Actinomycetes species isolated from the soil of Kazakhstan were cultured in modified media mimicking extreme environment the species were isolated from. Antimicrobial compound(s) extracted with organic solvent were tested against conditionally pathogenic and multi-drug resistant pathogens Acinetobacter baumanni and Pseudomonas aeruginosa.

Our study generated promising results regarding the potential discovery of novel components effective against drug resistant pathogens. Future studies will focus on further chemical analysis to identify the active component within these extremophilic extracts.
\end{abstract}

\section{Introduction}

Antibiotics, first time discovered in 1928 by Alexander Fleming, ${ }^{1}$ have become excellent chemotherapeutic agents effective in treating human infections caused by many dangerous pathogenic bacteria. Even though it was unexpectedly discovered, antibiotics and search for newer more effective antimicrobial agents brought science and medicine to an extensive research in this field. As a result, many more antibiotics, such as vancomycin, tetracycline, ampicillin and others were discovered within a short period of time. ${ }^{2}$

Natural sources of antibiotics can be bacteria and fungi. The most prevalent antibiotic producers are actinomycetes species, which are gram-positive fungi-like bacteria. Today $45 \%$ of all known antibiotics are produced by these species which are generally found in soil and freshwater. ${ }^{3}$ Most of the actinomycetes grow in neutrophilic and slightly alkaline conditions. However, there are also acidophilic species that grow in the $\mathrm{pH}$ range of $3.5-6.5$ with salt concentrations that are above $0.2 \mathrm{~mol} / \mathrm{L}$ $\mathrm{NaCl} .{ }^{4}$ These microorganisms are usually found in saline soil and are very promising targets for antimicrobial agents. The search for these organisms as producers of novel antibiotics is the subject of our current research.

We have found that there are actinomycetes species inhabiting soil with high salt and high alkaline concentration; these species appeared to produce antibiotics while growing in saline, alkaline, or neutral conditions. ${ }^{5}$ We continue our efforts in researching extremophilic actinomycetes that can be isolated from these extreme environmental growth conditions and screen their activity against multi-drug resistant bacteria that can cause life-threatening infections.

Bacterial ESKAPE pathogens, which are Enterococcus faecium, Staphylococcus aureus, Klebsiella pneumoniae, Acinetobacter baumannii, Pseudomonas aeruginosa, and Enterobacter spp., are recognized as Multi-Drug Resistant (MDR) microorganisms. These ESKAPE pathogens are resistant to almost all known classes of antibiotics and can cause healthcare associated infections (HAI). ${ }^{6}$ It is estimated that by 2050 infections caused by HAI pathogens with Antimicrobial Resistance (AMR) will cause fatality in up to 10 million people per year. ${ }^{7}$ Currently effective treatment against these drug resistant pathogens is scarce or nonexistent in some cases, making the situation even more serious. Thus, the search for novel antibacterial compounds that are effective against these multi-drug resistant pathogenic bacteria becomes even more crucial in this era of
Correspondence: Azliyati Azizan, Nazarbayev University School of Medicine (NUSOM), 5/1 Kerey and Zhanibek Khans Street, NurSultan, 010000, Republic of Kazakhstan

E-mail: azliyati.azizan@nu.edu.kz Tel.: +7-7172- 69-4633

Key words: Extremophiles; antibiotics; natural products; ESKAPE pathogens.

Acknowledgements: The authors would like to acknowledge the Research and Production Center for Microbiology and Virology, as well as the Republican Diagnostic Center for supporting us through this project. We also want to thank Nazarbayev University for funding this research.

Contributions: AZ performed microbiolgical part of the study; ZK performed chemical analysis on analytical HPLC, adjusted parameters, and designed a method for analysis; SS, ZM, DA performed molecular analysis including primer selection, PCR, and sequencing; LT provided the extremomophilic actinomycetes from her collection, and guided the team throughout the project; AA supervised the project, provided assistance to all group members, manuscript writing and checking.

Funding: the funding was provided by Nazarbayev University (NU), grant no. SOM2017001.

Conference presentations: Part of this paper was presented at the American Society for Microbiology Microbe 2018 Conference, Atlanta, Georgia, USA, in June 7-11, 2018.

Conflict of interest: The authors declare no potential conflict of interests.

Received for publication: 29 October 2019. Accepted for publication: 3 January 2020.

This work is licensed under a Creative Commons Attribution NonCommercial 4.0 License (CC BY-NC 4.0).

(C) Copyright: the Author(s), 2020

Licensee PAGEPress, Italy

Microbiology Research 2020; 11:8356

doi:10.4081/mr.2020.8356

diminishing treatment options.

Even though most of the antibiotics have been rediscovered, which led to the decline in drug discovery research activities, there is still hope that nature has solutions to the health problems that we have today. Antibiotics from natural sources usually are small compounds that are responsible for the inhibitory activity against other bacteria. However, synthesis of these naturally occurring compounds in the laboratory is difficult because of the complexity of the compound structure. Chemical analysis is 
required to elucidate the exact component within the natural products that is responsible for the activity against these pathogens.

Most of the research on screening antibiotic compounds from natural products target prokaryotic organisms from the phylum actinomycetes extracted from neutral environment. Interestingly, actinomycetes are also one of the most widespread extremophiles that can grow in harsh growth conditions and they can maintain favorable conditions for themselves. These microorganisms produce antibiotics naturally as a defense mechanism in order to survive and thrive in their natural environments. Thus, it is reasonable to investigate antibiotic producing potential of these microorganisms by isolating and growing them in extreme laboratory culture conditions that would mimic their natural growth environment.

We found that extremophilic actinomycetes that were isolated from the saline soil were able to inhibit growth of other bacteria including our tested MDR organisms. ${ }^{8}$ Moreover, they produce inhibitory antibiotics only in response to specific growth conditions. Thus, salt for example can be the trigger for actinomycetes to produce antimicrobial compound. Identification of the compound associated with the inhibitory antibacterial activity was performed using High-Performance Liquid Chromatography (HPLC); this was approached by analyzing and comparing differential peaks between extracts from the same strain grown in different culture conditions. We hypothesize that extremophiles grown in different extreme growth conditions (either saline or alkaline) could produce antimicrobial compounds that have not been yet identified and investigated, and therefore are good candidates of microorganisms that could produce novel antibacterial compounds effective against MDR bacterial pathogens.

\section{Materials and Methods}

\section{Overall study design}

This study was a continuation of an ongoing research as we have previously reported. ${ }^{8}$ Our study found that actinomycetes species are capable of producing antimicrobial activity when these are grown in modified growth media. All the strains used in this study were isolated from extreme environments of Kazakhstan. The strains were isolated from environmental samples collected, which were soils, muds, and rhizosphere samples from Northern and Southern Kazakhstan. Samples collected were transferred to the laboratory for isolation of extremophilic microorganisms which were then grown and extracted to obtain the source of the inhibitory antibiotic for testing against bacterial pathogens. This step was then followed by chemical characterization of active components using HPLC analysis, and finally molecular analysis for identification of the strains.

\section{Subject, sampling, recruitment}

Soil samples from deserts, solonchaks and forests of Ile-Balkhash and Amankaragay regions in Kazakhstan were collected to obtain the 5 extremophilic actinomycete strains number 9, 78, 334, 536, and 550 that are described in this study, as potential producers of novel antibiotics (Figure 1).

\section{Isolation and maintenance of Actinomycetes}

Five strains of actinomycetes species (strains \#9, 78, 334, 536, and 550) were
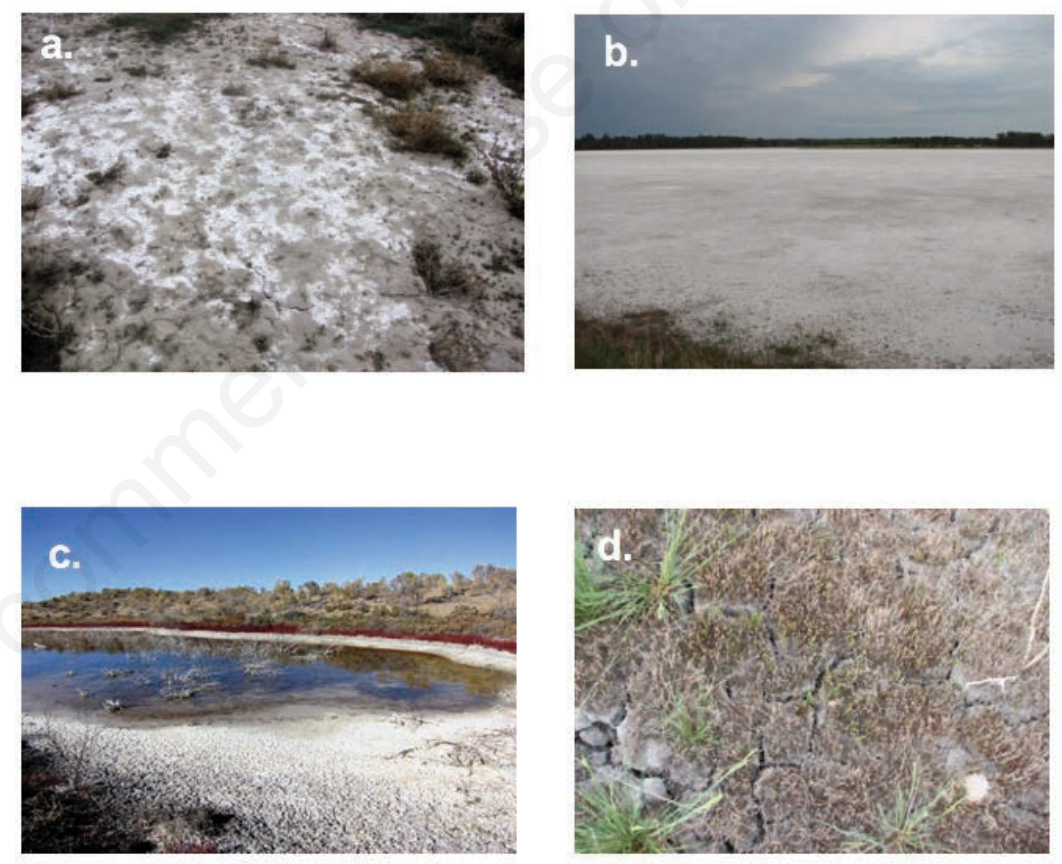

grown in four different modified Bennett's broth media, which were neutral, saline 1 , saline 2 and alkaline. Neutral medium was composed of Oatmeal flour $(1.5 \mathrm{~g}), \mathrm{CaCO}_{3}$ $(0.25 \mathrm{~g})$, and Glucose $(1.5 \mathrm{~g})$ in a total growth media volume of $100 \mathrm{ml}$ per flask (strains grown in triplicate flasks/strain/ medium). Saline 1 medium was prepared similarly as neutral media but with the addition of $2.5 \mathrm{~g}$ of salt, while Saline 2 medium contained $5 \mathrm{~g}$ of salt, and alkaline medium contained $0.5 \mathrm{~g}$ of $\mathrm{Na}_{2} \mathrm{CO}_{3}$ solution. This growth culture condition was designed to mimic natural environment from where these bacteria were obtained. Actinomycetes were grown for 5 days at $28^{\circ} \mathrm{C}$ with sufficient aeration (continuous shaking in a large $1000 \mathrm{ml}$ flask) for optimal growth, after which extraction was performed using organic solvent. This protocol was described in our previous research; ${ }^{9}$ however, in this study n-butanol was used to

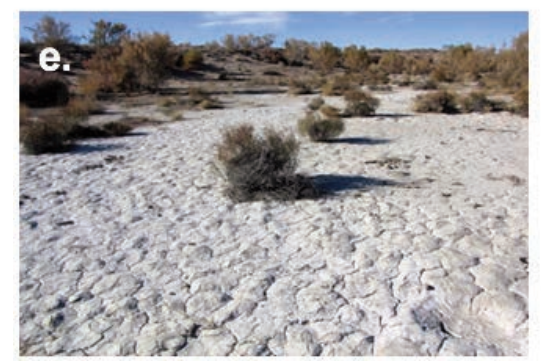

Figure 1. Sampling sites for Actinomycetes strains \#9 (a), 78 (b), 334 (c), 536 (d), and 550 (e). a. Ile-Balkhash region, clay desert, salty soil; b. Amankaragay pine forest, saline solonchak, a sample of the rhizosphere; c. Ile-Balkhash region, sandy desert, saline solonchak; d. Amankaragay pine forest, saline solonchak, a sample of the rhizosphere; e. IleBalkhash region, sandy desert, typical solonchak. 
extract the antibiotic from the cultural liquid of actinomycetes broth (instead of ethyl acetate that was previously used as a solvent). Culture media from 3 flasks of each strain grown in same medium were combined and filtered through the Büchner funnel and a cloth. By doing this biomass and culture liquid were divided and used separately for extraction. Volume of the culture broth was measured and transferred to a separating funnel. N-butanol was added in a 1:5 ratio to each funnel with the culture liquid and mixed well periodically during the day and left to stay overnight under the fume hood, so that two phases (organic and inorganic) separate. Organic (top) phase was collected to the clean vial and further evaporated in the rotary evaporator at 14 $\mathrm{mmHg}$ pressure at $40^{\circ} \mathrm{C}$. Finally, the evaporated dry extracts were dissolved in $90 \%$ ethanol and stored at $4^{\circ} \mathrm{C}$ for future analysis.

Extraction from biomass (cell pellet) was also performed to obtain the antibiotic compound left within the cells. Pellet left after filtration of the actinomycetes growth media was dried wrapped in the clean cloth and filter paper. After drying, the biomass was transferred to a clean vial, and mixed with $90 \%$ ethanol in $1 \mathrm{~g}: 3 \mathrm{ml}$ ratio, and left overnight at $4^{\circ} \mathrm{C}$. Next day, the biomass was filtered out and liquid extract was evaporated in the rotary evaporator at $97 \mathrm{mmHg}$ pressure at $40^{\circ} \mathrm{C}$. The solvent was evaporated up to the volume equal to $2 / 100$ times the volume of the filtrate (culture liquid), and stored at $4^{\circ} \mathrm{C}$. Each extract was labeled and given the numbers 1 and 2, which defines the origin of the extract, either from culture liquid (designated as 1) or biomass (designated as 2), respectively. For instance, extract \#9-1 represents the extract from strain \#9 extracted from culture liquid, and extract \#9-2 is the extract obtained from the biomass of strain \#9. The flowchart summarizing this method to obtain "crude extracts" used in the assays described below for antimicrobial activity and HPLC is shown in Figure 2.

\section{In vitro antimicrobial assay}

Three levels of screening of the extract antibacterial activities were performed. The first level of screening was done after separation of culture liquid and biomass. Approximately 1-2 $\mathrm{ml}$ of culture liquid was taken for analysis before addition of nbutanol, and sample from biomass was taken after the addition of ethanol and before evaporation. The second level of screening was performed using the final product (which were the extracts obtained after evaporation and reconstitution). The procedure for both screenings is the same except that the samples tested are different. For the first and second levels of screen- ings, we tested the inhibitory activity of samples against conditionally pathogenic bacteria Staphylococcus aureus and Escherichia coli. For the third level of screening we performed testing on hospital strains of MDR pathogens, which included Acinetobacter baumannii and Pseudomonas aeruginosa. These hospital pathogens were provided by the Republican Diagnostic Center in Nur-Sultan, Kazakhstan. Inhibitory antibacterial activity was determined using the standard disc diffusion and wells assay methods. The standard disc diffusion assay was performed by applying 20 $\mu \mathrm{L}$ of the extract to the sterile disc and testing this along with the control antibiotics discs. Solutions containing the appropriate amount of test organisms in growth media were prepared according to $0.5 \mathrm{Mc}$ Farland standard. ${ }^{10} 200 \mu$ l of suspension was pipetted onto the surface of Mueller-Hinton agar in Petri dishes and spread with a sterile spreader over the whole surface of the agar; thereafter extract-containing discs were placed onto the agar surface containing the test microorganisms. Placement of disc were performed clockwise from a starting point, beginning with the extracts that were grown in neutral, then saline 1 , saline 2 , and alkaline media, accordingly. We also performed the wells assay method as an alternative method to compare the inhibitory activities of the extracts to enable us to compare with the results of the disc diffusion assay. The advantage of this method was that more quantity of the extracts could be applied to the wells (up to $100 \mu \mathrm{l}$, compared to $20 \mu \mathrm{l}$ total volume possible for the disc method). For the wells method, liquid nutrient agar media was seeded with a solution of $10^{9} \mathrm{CFU}$ (colony forming unit) of the test microorganisms and this mixture was then poured into the petri dishes. After solidification of the agar, four $7 \mathrm{~mm}$-diameter wells were created using a cork borer. Finally, $0.1 \mathrm{ml}$ of each sample was pipetted into these wells clockwise starting from neutral media, saline 1 , saline 2 , and alkaline. This was done for extracts from both the filtrate and the biomass. Inoculated petri dishes were incubated for one day, after which the activities (zones of inhibition) were measured.

\section{Chemical characterization}

Analytical high-performance liquid chromatography was used for initial analysis of differences between the extracts with antibiotic activity and those without activity. To separate the content of the extracts, reverse phase $\mathrm{C} 18$ column with increasing acetonitrile gradient was used. $20 \mu \mathrm{l}$ of sample was used per run and detected at $210 \mathrm{~nm}$ wavelength.

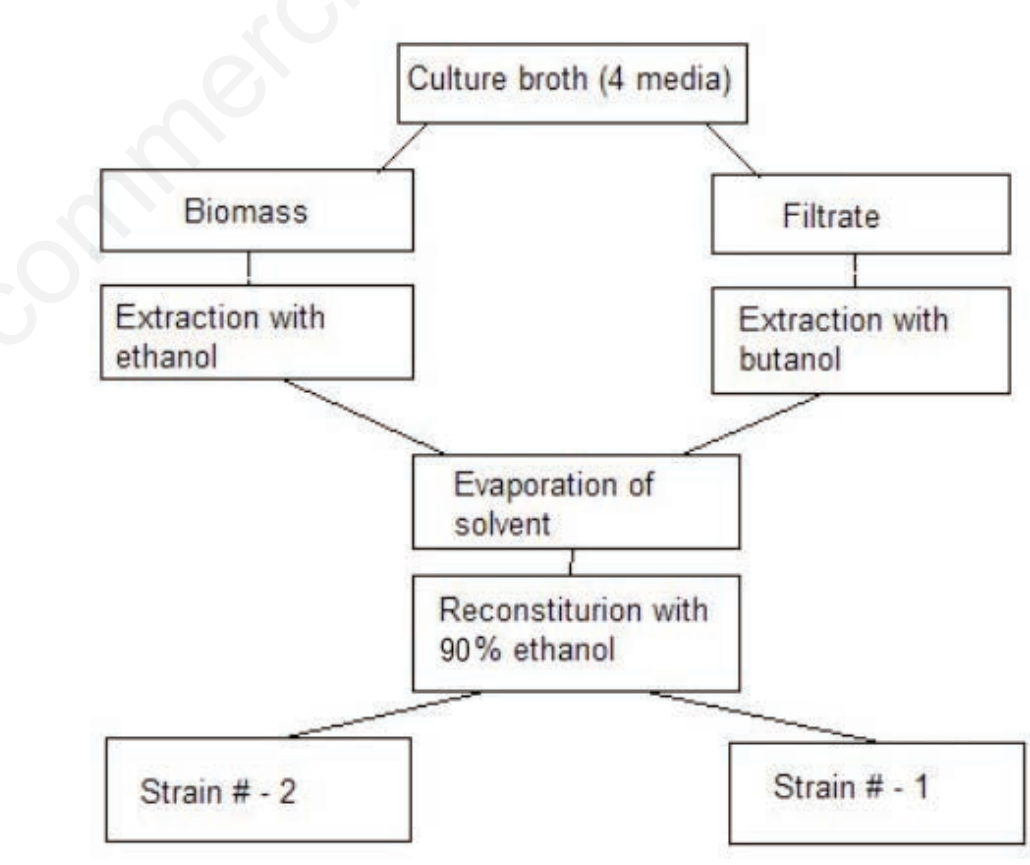

Figure 2. Flowchart for the extraction of antibiotic method from actinomycetes species. Strains were cultured in broth media, then grown culture was filtered through the Büchner funnel with a cloth inside. So that the biomass can stay on the cloth. Afterwards, organic phase of a culture liquid was extracted and separated by addition of $\mathbf{n}$-butanol in a separatory funnel. Biomass was dried and suspended in $90 \%$ ethanol overnight. Finally, solvents (n-butanol and ethanol) were evaporated in the rotary evaporator and resuspended in $90 \%$ ethanol to a final volume of $\sim 2-3-\mathrm{ml}$. Extracts were then given numbers -1 or -2 , which defines the origin of the extract, culture liquid or biomass, respectively. 
machine and blast search analysis was performed to identify the strains of actinomycetes. tify the 5 actinomycetes strains, PCR was performed using the primers TCACGGA-

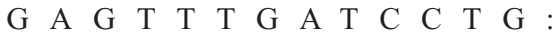
(forward rRNA actinomycetes) and GCGGCTGCTGGCACGTAGTT (reverse rRN

A actinomycetes) as previously reported, ${ }^{11}$ on Bio-Rad T100 thermal cycler machine. The PCR conditions used were; initial denaturation at $96^{\circ} \mathrm{C}, 5 \mathrm{~min}$; denaturation $96^{\circ} \mathrm{C}, 1 \mathrm{~min}$; annealing $48^{\circ} \mathrm{C}, 1 \mathrm{~min}$; extension $68^{\circ} \mathrm{C}, 1 \mathrm{~min}$, for 40 cycles; and final extension at $68^{\circ} \mathrm{C}, 5 \mathrm{~min}$. The total PCR product volume was $20 \mu \mathrm{l}$. The PCR products were then sent for sequencing analysis to National Center for Biotechnology (NCB) in Nur-Sultan, Kazakhstan. Sequencing was performed according to standard protocol using 3730XL Applied Biosystems sequencing

\section{Results}

Extracts obtained from culture media and the biomass showed inhibitory antibacterial activity. Most of the extracts were either only active when grown in saline media, or had better inhibitory activity against the selected test bacteria, when grown with the increased $\mathrm{pH}$ growth conditions. Preliminary chemical analysis showed comparable chromatograms, which correlate with the activity of the extracts. All of the five strains were sequenced and appeared to be actinomycetes species, specifically belonging to the Streptomyces and Nocardiopsis genera.

\section{Disc diffusion and wells assay}

Wells assay showed significant inhibitory activity of extracts against the conditionally pathogenic bacteria $E$. coli and $S$. aureus. Results of secondary levels of screening are shown in Table 1. Extracts from strain \#334 grown in saline media showed inhibition of both test microorganisms, whereas extracts from the strain grown in neutral and alkaline media did not exhibit antimicrobial activity. Extracts from culture broth of strains \#536 and 550 grown in all four media conditions showed activity against $S$. aureus. Extract \#78 was effective against all five test microorganisms whereas extract \#9 had minor inhibition of $S$. aureus in alkaline medium. Results of the third level of screening of these extracts performed on the MDR pathogens $A$. baumannii and $P$. aeruginosa are summarized in Table 2. Antibiogram for test bacterial

Table 1. Secondary screening using wells assay. Tests were done in duplicates. Average ZOI in mm is shown in table.

\begin{tabular}{llcccc} 
Strain \# & Microorganism & Neutral medium $(\mathrm{mm})$ & Saline 1 medium $(\mathrm{mm})$ & Saline 2 medium $(\mathrm{mm})$ Alkaline medium (mm) \\
\multirow{2}{*}{$9-2$} & S. aureus & 0 & 0 & 0 & $14 \mathrm{bs}$ \\
& E. coli & 0 & 0 & 0 & 0 \\
$78-1$ & S. aureus & $9 \mathrm{bc}$ & $10 \mathrm{bc}$ & $12 \mathrm{bs}$ & 0 \\
& E. coli & $13 \mathrm{bc}$ & $10 \mathrm{bc}$ & $12 \mathrm{bc}$ & $11 \mathrm{bc}$ \\
\hline $334-2$ & S. aureus & 0 & $10 \mathrm{bc}$ & $13 \mathrm{bc}$ & 0 \\
& E. coli & 0 & $24 \mathrm{bc}$ & $12 \mathrm{bc}$ & 0 \\
$536-1$ & S. aureus & $13 \mathrm{bc}$ & $14 \mathrm{bc}$ & $18 \mathrm{bc}$ & $15 \mathrm{bc}$ \\
& E. coli & 0 & 0 & 0 & 0 \\
\hline $536-2$ & S. aureus & $9 \mathrm{bc}$ & $12 \mathrm{bc}$ & 0 & 0 \\
& E. coli & 0 & 0 & $12 \mathrm{bs}$ & $20 \mathrm{bs}$ \\
$550-1$ & S. aureus & $12 \mathrm{bs}$ & $16 \mathrm{bs}$ & $13 \mathrm{bc}$ & $12 \mathrm{bc}$ \\
& E. coli & 0 & $11 \mathrm{bc}$ &
\end{tabular}

ZOI - zone of inhibition. bc - bactericidal effect (completely clear zones without any growth of test-microorganism). bs - bacteriostatic effect (slightly opaque zone or small colonies of test-microorganism grown within the zone of inhibition).

Table 2. Inhibition zones from activity test using wells method against pathogens. Tests were done in duplicates. Average ZOI in mm is shown in table.

Strain \# Microorganism Neutral medium ( $\mathrm{mm}$ ) Saline 1 medium ( $\mathrm{mm}$ ) Saline 2 medium (mm) Alkaline medium (

\begin{tabular}{|c|c|c|c|c|c|}
\hline $9-2$ & $\begin{array}{l}\text { A. baumannii } \\
\text { P. aeruginosa }(\mathrm{S}) \\
\text { P. aeruginosa }(\mathrm{R}\end{array}$ & $\begin{array}{c}5.5 \mathrm{bc} \\
0 \\
0\end{array}$ & $\begin{array}{l}0 \\
0 \\
0\end{array}$ & $\begin{array}{c}0 \\
10 \mathrm{bc} \\
0\end{array}$ & $\begin{array}{l}0 \\
0 \\
0\end{array}$ \\
\hline 78-1 & $\begin{array}{l}\text { A. baumannii } \\
\text { P. aeruginosa }(\mathrm{S}) \\
\text { P. aeruginosa }(\mathrm{R})\end{array}$ & $\begin{array}{l}9.5 \mathrm{bc} \\
10 \mathrm{bc} \\
13.5 \mathrm{bc}\end{array}$ & $\begin{array}{l}8.5 \mathrm{bc} \\
10 \mathrm{bc} \\
11 \mathrm{bc}\end{array}$ & $\begin{array}{c}0 \\
12 b c \\
10 b c\end{array}$ & $\begin{array}{c}0 \\
11.5 \mathrm{bc} \\
10.5\end{array}$ \\
\hline 78-2 & $\begin{array}{l}\text { A. baumannii } \\
\text { P. aeruginosa (S) } \\
\text { P. aeruginosa }(\mathrm{R})\end{array}$ & $\begin{array}{c}0 \\
9 \mathrm{bc} \\
11 \mathrm{bc}\end{array}$ & $\begin{array}{c}0 \\
9.5 \mathrm{bc} \\
10 \mathrm{bc}\end{array}$ & $\begin{array}{c}0 \\
9 \mathrm{bc} \\
13 \mathrm{bc}\end{array}$ & $\begin{array}{c}0 \\
10 \mathrm{bc} \\
12 \mathrm{bc}\end{array}$ \\
\hline $536-2$ & $\begin{array}{l}\text { A. baumannii } \\
\text { P. aeruginosa }(\mathrm{S}) \\
\text { P. aeruginosa }(\mathrm{R})\end{array}$ & $\begin{array}{l}0 \\
0 \\
0\end{array}$ & $\begin{array}{c}0 \\
10 \mathrm{bc} \\
0\end{array}$ & $\begin{array}{c}0 \\
11 \mathrm{bc} \\
0\end{array}$ & $\begin{array}{c}0 \\
10 \mathrm{bc} \\
0\end{array}$ \\
\hline $550-1$ & $\begin{array}{l}\text { A. baumannii } \\
\text { P. aeruginosa (S) } \\
\text { P. aeruginosa }(\mathrm{R})\end{array}$ & $\begin{array}{c}9.5 \mathrm{bc} \\
11 \mathrm{bc} \\
0\end{array}$ & $\begin{array}{c}10 \mathrm{bc} \\
11.5 \mathrm{bc} \\
0\end{array}$ & $\begin{array}{c}10 \mathrm{bc} \\
11 \mathrm{bc} \\
0\end{array}$ & $\begin{array}{c}0 \\
10.5 \mathrm{bc} \\
0\end{array}$ \\
\hline $550-2$ & $\begin{array}{l}\text { A. baumannii } \\
\text { P. aeruginosa (S) } \\
\text { P. aeruginosa }(\mathrm{R})\end{array}$ & $\begin{array}{c}4 \mathrm{bc} \\
11.5 \mathrm{bc} \\
0\end{array}$ & $\begin{array}{c}4 \mathrm{bc} \\
4 \mathrm{bc} \\
0\end{array}$ & $\begin{array}{c}4 \mathrm{bc} \\
9.5 \mathrm{bc} \\
0\end{array}$ & $\begin{array}{c}4 \mathrm{bc} \\
10.5 \mathrm{bc} \\
0\end{array}$ \\
\hline
\end{tabular}

ZOI - zone of inhibition. bc - bactericidal effect (completely clear zones without any growth of test-microorganism). bs - bacteriostatic effect (slightly opaque zone or small colonies of test-microorganism grown within the zone of inhibition) 
pathogens tested against standard antibiotics is shown in Table 3. For P. aeruginosa we tested our extracts against the drug sensitive as well as drug resistant strains. Extract \#78-1 inhibited MDR resistant pathogens A. baumannii and P. aeruginosa, exhibiting bactericidal effect. Extracts \# 781, 78-2, 536-1, 550-1, and 550-2 all had activity against drug-sensitive $P$. aeruginosa. Extract \#78-2 had promising effect against drug-resistant $P$. aeruginosa. Extracts from strain \#550 had a weak yet promising activity against MDR resistant $A$. baumannii. Extracts \#334-1 and 334-2 unfortunately, did not show any inhibition of MDR pathogens. All of the extracts showed clear zones, which suggests that they have completely killed the pathogen around the discs/wells, having bactericidal effect.

\section{High-Performance Liquid Chromatography (HPLC)}

HPLC analysis showed the peaks which can be correlated with the activity of the extract. Comparing the extracts derived from the same strain of actinomycetes but grown in different media, we were able to identify differential peaks that might be associated with the antibacterial inhibitory activities of the extracts. For example, the extract \#334-2, which had activity in saline

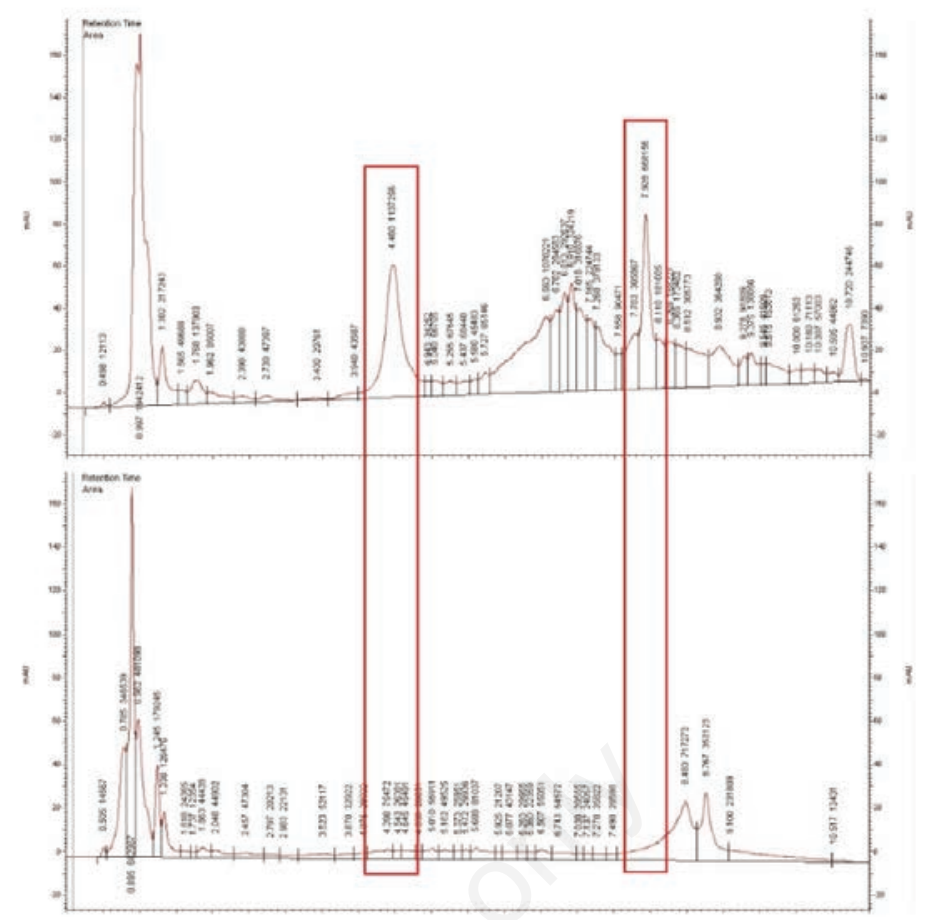

Figure 3. HPLC chromatogram for extract \#334-2. A. Saline 2 media (50g salt); B. Alkaline media. There are notable peaks in Saline 2 media which also had activity against conditionally pathogenic bacteria $S$. aureus and $E$. coli, as well as resistant $P$. aeruginosa. Whereas the extract grown in alkaline media did not have activity in any test and, consistently, the chromatogram does not have same peaks.

Table 3. Antibiogram for Acinetobacter baumannii and Pseudomonas aeruginosa test microorganisms.

\begin{tabular}{|c|c|c|c|}
\hline Antibiotics & $\begin{array}{c}\text { Acinetobacter } \\
\text { baumanit MRGN }\end{array}$ & $\begin{array}{l}\text { Name of the microorganisms } \\
\text { Pseudomonas aeruginosa MRGN } \\
\text { Sensitivity of microorganisms }\end{array}$ & $\begin{array}{c}\text { Pseudomonas } \\
\text { aeruginosa }\end{array}$ \\
\hline
\end{tabular}

Ampicillin sulbactam

$$
\mathrm{R}
$$

\begin{tabular}{llll} 
Cephtazidime & $\mathrm{R}$ & $\mathrm{R}$ & $\mathrm{S}$ \\
\hline Imipenem & $\mathrm{R}$ & $\mathrm{R}$ & $\mathrm{S}$ \\
Meropenem & $\mathrm{R}$ & $\mathrm{R}$ & $\mathrm{S}$ \\
\hline Gentamycin & $\mathrm{R}$ & $\mathrm{R}$ & $\mathrm{S}$ \\
Ticarcillin & & $\mathrm{R}$ & $\mathrm{S}$ \\
\hline Ciprofloxacin & $\mathrm{R}$ & $\mathrm{R}$ & $\mathrm{S}$ \\
Levofloxacin & $\mathrm{R}$ & $\mathrm{R}$ & $\mathrm{S}$ \\
\hline Piperacillin & $\mathrm{R}$ & $\mathrm{R}$ & $\mathrm{S}$ \\
Piperacillin/ tazobactam & & $\mathrm{S}$ \\
Ticarcillin-clavulanate & $\mathrm{R}$ & $\mathrm{R}$ & $\mathrm{S}$ \\
Cefepime & $\mathrm{R}$ & & \\
\hline Cefotaxime & $\mathrm{R}$ & $\mathrm{R}$ & $\mathrm{S}$ \\
Ceftriaxone & $\mathrm{R}$ & $\mathrm{R}$ & \\
\hline Aztreonam & & $\mathrm{R}$ & \\
Amikacin & $\mathrm{S}$ & & \\
Tetracycline & $\mathrm{R}$ & $\mathrm{S}$ \\
Doxycycline & $\mathrm{R}$ & $\mathrm{R}$ & \\
\hline Trimetoprime/ sufometaxol & $\mathrm{R}$ & & \\
\hline
\end{tabular}

MRGN - multi-resistant Gram-Negative resistance to 4 types of antibiotics: ureapenicillins, 3-4 generation cefasporins, cabrophenes, fluoroquinolones. S- sensitive. R- resistant. A. baumannii and P. aeruginosa labeled as MRGN are resistant to 4 classes of antibiotics. One strain of P. aeruginosa is sensitive to all classes of antibiotics. This strains were taken for comparison with resistant test microorganisms, and see the potential of our crude extract. 
1 medium $(13 \mathrm{~mm})$ and did not have the activity in alkaline medium $(0 \mathrm{~mm})$ had distinguishable chromatograms with different peaks (boxed) (Figure 3). Extract \#9-2 inhibited $S$. aureus in alkaline medium, whereas in other media there was no effect. Correspondingly in Figure 4 we can see that chromatogram of the extract in alkaline environment has a significant peak, whereas in extract from saline 2 medium there is a plateau at the same time point (boxed). Chromatograms of the extract \#536-1 both have different contents in them (Figure 5); as shown in Table 1, secondary screening of this extract showed antibacterial activity in both saline and alkaline media against $S$. aureus.

\section{Sequencing data}

Sequencing data was obtained as an alignment of nucleotide sequence for each strain. Basic Local Alignment Search Tool (BLAST) was used to find similarity of obtained sequences to those present in the database. Three of five strains (strain \#9, 78 , and 334) had 98 to $100 \%$ similarity to Streptomyces species. Two strains (\#536 and 550) had similarity to Nocardiopsis species. Most of the strains associated with our actinomycetes strains have antibiotic activity that were previously reported (Table 4).

\section{Discussion}

Culture conditions for growing actinomycetes influence the production of bacterial inhibitory compounds by extremophilic actinomycetes. This was illustrated in the HPLC chromatogram that showed correlation of specific peaks to the antibacterial inhibitory activity tests (disc diffusion and wells assay) that were performed in this study. Extremophilic actinomycetes can potentially be the bacterial species that produce antibacterial agents that have not been previously identified and reported.

It has been long known that scientists should come up with a new strategy to find new antibiotics compound and not re-discover known ones. Therefore, it is reasonable to try finding new sources of antibiotics using alternative methods to the traditional approaches. Until now actinomycetes species have been the most abundant natural source that was found to produce antibiotic. However recently, the discovery of novel antibiotics from these organisms has waned. Since it has been found that actinomycetes can also live in extreme environments such as at high saline, high alkaline and temperature conditions, our research focus has been to screen for antibiotic producing organisms from those extremophiles

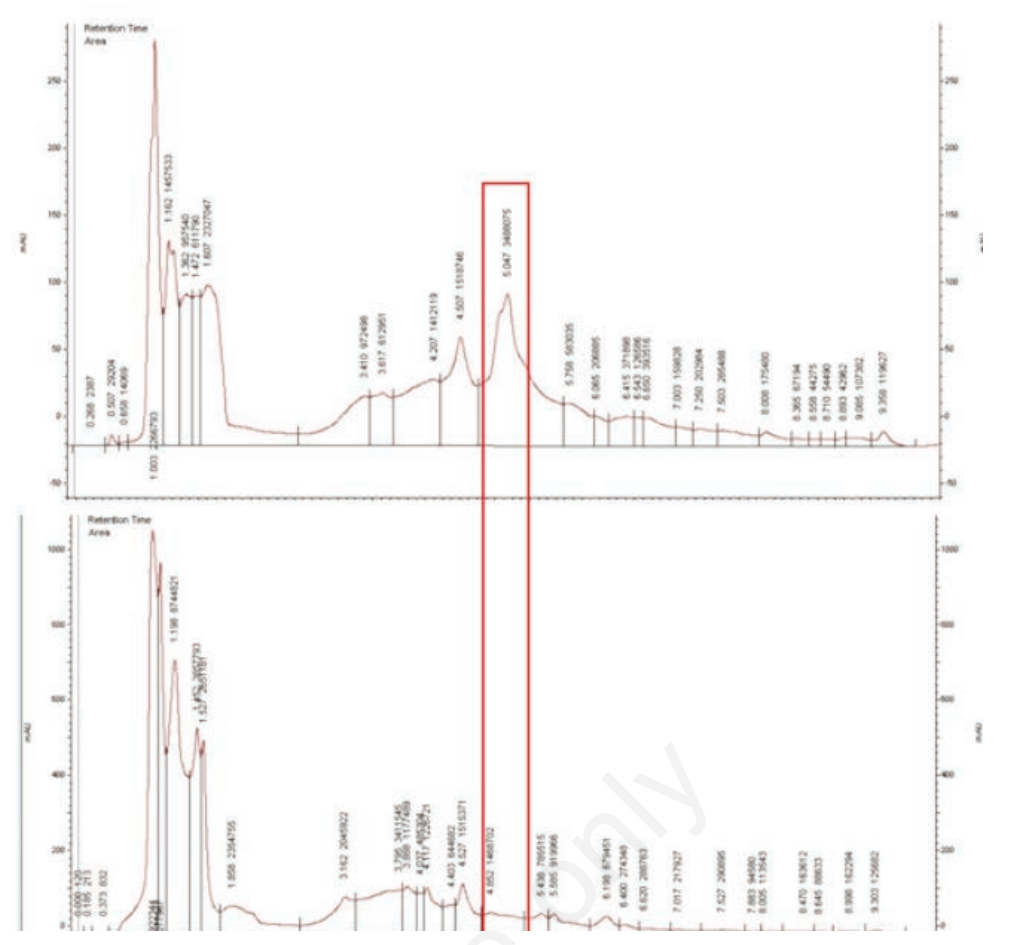

Figure 4. HPLC chromatogram for extract \#9-2. A. Saline 2 medium (50g salt); B. Alkaline medium. Chromatograms differ according to the media they come from. Saline 2 medium has significant peaks compared to the alkaline medium. This correlates with the inhibitory activity against $S$. aureus, $0 \mathrm{~mm}$ in saline medium, and $14 \mathrm{~mm}$ in alkaline medium. 
instead of just actinomycetes isolated from neutral environments, using specialized culture conditions that mimic the original environment. It was found that in such extreme conditions, Actinobacteria are able to maintain favorable environment for themselves and inhibit growth of other bacteria by producing special compounds. Halophilic and alkaliphilic actinomycetes are being consid- ered a potential source of novel antibiotics. Different studies have found such compounds as abenquines, chaxalactins, and others from actinomycetes isolated from deserts and arid habitats. ${ }^{12}$ As such, Ramya et al. (2015), ${ }^{13}$ investigated the property of actinomycetes to produce selenium nanoparticles extracellularly, which have anti-biofilm, anti-cancer, and anti-viral activities. All of these studies show that extreme environments, being desert, marine ecosystems, or mud, contain unique actinobacteria that still can produce antimicrobial, possibly novel, compounds. However, the strategy of these studies only directed to isolate the bacteria from extreme environments, but not grow them in similar environments. To that end, the approach used in

\section{Table 4. Sequencing results to elucidate actinomycetes strain identity}

\begin{tabular}{|c|c|c|c|c|}
\hline Strain \# & Strain BLAST search match & Strain species & Presence in genome & Reported antibiotic activity \\
\hline 9 & Streptomyces sp. strain & $\begin{array}{l}\text { Streptomyces halotolerans } \\
\text { Streptomyces daliensis } \\
\text { Streptomyces luteoverticillatus } \\
\text { Kitasatospora aureofaciens } \\
\text { Streptomyces rimosus strain } \\
\text { Streptomyces tacrolimicus }\end{array}$ & $\begin{array}{l}99 \% \\
98 \% \\
98 \% \\
98 \% \\
98 \% \\
98 \%\end{array}$ & $\begin{array}{l}\text { - Has antimicrobial activity against Bacillus } \\
\text { subtilis (ACCC 11060), Staphylococcus } \\
\text { aureus (AS 1.72), Micrococcus luteus (ACCC } \\
\text { 11001), 'Sarcinalutea' (AS 1.241) and } \\
\text { Xanthomonas oryzae (AS 1.843). }{ }^{14,16} \\
\text { - N/A } \\
\text { - N/A } \\
\text { - N/A } \\
\text { - N/A } \\
\text { - } \text { Oxytetracycline and other tetracycline } \\
\text { antibiotics. }{ }^{15} \\
\text { - N/A }\end{array}$ \\
\hline 78 & Streptomyces sp. strain & $\begin{array}{l}\text { Streptomyces pratensis } \\
\text { Streptomyces lavendulae } \\
\text { Streptomyces cyaneofuscatus } \\
\text { Streptomyces griseoplanus } \\
\text { Streptomyces fimicarius } \\
\text { Streptomyces flavogriseus }\end{array}$ & $\begin{array}{l}100 \% \\
100 \% \\
100 \% \\
98 \% \\
99 \% \\
98 \%\end{array}$ & 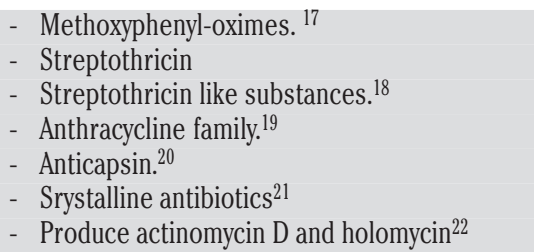 \\
\hline 334 & Streptomyces sp. strain & Streptomyces halotolerans strain & $99 \%$ & $\begin{array}{l}\text { - Has antimicrobial activity against Bacillus } \\
\text { subtilis (ACCC 11060), Staphylococcus } \\
\text { aureus (AS 1.72), Micrococcus luteus (ACCC } \\
\text { 11001), 'Sarcinalutea' (AS 1.241) and } \\
\text { Xanthomonas oryzae (AS 1.843) 14,16 } \\
\text { - N/A }\end{array}$ \\
\hline 536 & Nocardiopsis sp. strain & $\begin{array}{l}\text { Nocardiopsis dassonvillei } \\
\text { Nocardiopsis synnemataformans } \\
\text { Nocardiopsis alba }\end{array}$ & $\begin{array}{l}97 \% \\
98 \%\end{array}$ & $\begin{array}{l}\text { - Extracellular enzymes such as amylases, } \\
\text { chitinases, cellulases, } \beta \text {-glucanases, } \\
\text { inulinases, xylanases and proteases }{ }^{23} \\
\text { - } \text { N/A } \\
\text { - }\end{array}$ \\
\hline 550 & Nocardiopsis sp. strain & $\begin{array}{l}\text { Nocardiopsis terrae } \\
\text { Nocardiopsis dassonvillei }\end{array}$ & $\begin{array}{l}98 \% \\
99 \%\end{array}$ & $\begin{array}{l}\text { - Thiopeptide Antibiotic }{ }^{24} \\
\text { - Produce arabinose, cellobiose, fructose, } \\
\text { gluconate, glucose, mannose, maltose, } \\
\text { rhamnose, ribose, sucrose, trehalose, xylose, } \\
\text { mannitol, putrescine, acetate, cis-aconitate, } \\
\text { 4-aminobutyrate, azelate, citrate, fumarate, } \\
\text { glutarate, 3-hydroxybutyrate, pyruvate, } \\
\text { suberate, L-alanine, phenylalanine, proline, } \\
\text { serine, 4-hydroxybenzoate, and } \\
\text { phenylacetate, arbutin, galactose, melibiose, } \\
\text { salicin, adonitol, inositol, maltitol, sorbitol, } \\
\text { propionate, adipate, itaconate, lactate, } \\
\text { malate, mesaconate, oxoglutarate, } \beta \text {-alanine, } \\
\text { aspartate, histidine, leucine, ornithine, } \\
\text { tryptophan, or } 3 \text {-hydroxybenzoate, } \\
\text { phosphatase and } \alpha \text {-glucosidase }{ }^{25} \\
\text { - N/A } \\
\text { - Extracellular enzymes such as amylases, } \\
\text { chitinases, cellulases, } \beta \text {-glucanases, } \\
\text { inulinases, xylanases and proteases }{ }^{23}\end{array}$ \\
\hline
\end{tabular}

Actinomycetes strains \# 9, 78, and 334 that we used are Streptomycetes species, and strain \#536 and 550 are Nocardiopsis species. Strain \#78 has $100 \%$ similarity in its nucleotide sequence contents with Streptomyces pratensis, Streptomyces lavendulae, and Streptomyces cyaneofuscatus. Other strains do not have 100\% match in the sequence, which can suggest that this is a new actinomycete strain, not explored yet. The tables also include information about the known antibiotics that are associated with each microorganism that potentially can be our strain. Some of the strains, such as Streptomyces halotolerans, Streptomyces daliensis Streptomyces luteoverticillatus, Kitasatospora aureofaciens, Streptomyces tacrolimicus, Nocardiopsis synnemataformans, and Nocardiopsis terrae were not found to produce antibiotic compounds. 
our study was to use specific extreme culture media that mimic the organism's natural environment for growing the extremophilic actinomycetes species. Those strains such as the five chosen for this study were screened through various screening levels. After these strains were shown to consistently exhibit antibacterial properties against our test organisms, these were further characterized for active compound identification.

Extracts from strains \#78, 536, and 550, which showed the greatest inhibitory activity were classified according to the previous research as a group $\mathrm{I},{ }^{8}$ meaning that actinomycetes species are able to grow in all three conditions (neutral, saline, and alkaline). Based on the ability to show antagonism under different conditions, these three extracts are classified into following subgroups: extracts $\# 78$ and 550 are in subgroup IA and extract \# 536 in subgroup IBc. Both extracts \#78 and \# 550 show antagonism in three habitats, whereas extract \#536 show antagonism only in saline and alkaline habitats against tested ESKAPE pathogens. Even though the other extracts did not show very good inhibitory activity against hospital strains of MDR pathogens, these did show good antagonism against conditionally pathogenic $S$. aureus and $E$. coli. Classification of those extracts was as follows; extract \#334 belongs to subgroup $\mathrm{ICb}$ and extract \#9 in subgroup IBa. Mostly all the extracts had antagonistic effect in two habitats, whereby the saline environment shows the most inhibitions among all the extracts prepared from the strains included in this study.

Extract \#9-2 had only activity in alkaline environment (Table 1), and correspondingly HPLC chromatogram showed interesting contents in the same medium, but not in others (Figure 4). It is possible that the peak that showed up in the chromatogram is the compound that actually inhibits the growth of pathogenic bacteria, and it is only produced under specific alkaline environment. The same trend can be seen in extract \#334-2 (Figure 3). However, this strain favors saline environment for production of antibiotic compound, which can be seen both in the chromatogram (two clear peaks) and the antimicrobial activity (Table 1). Interestingly, extract \#536-1 inhibited growth of conditionally pathogenic $S$. aureus in all four media. But the chromatograms of this extract were substantially different compared to each other (Figure 5). This can suggest that different contents are released from bacteria depending on the medium they live in.

Sequencing results display that most of the matched species were previously reported to produce antibiotics as described below. For example, Streptomyces sparcus, the sequence of which is similar to our strains \#9 and 334, have been reported to have antibacterial activities against Bacillus subtilis (ACCC 11060), Staphylococcus aureus (AS 1.72), Micrococcus luteus (ACCC 11001), Sarcinalutea (AS 1.241) and Xanthomonas oryzae (AS 1.843). ${ }^{14}$ Streptomyces rimosus, which sequence was similar to our strain \#9 is very well-characterized species that is used to produce industrial tetracycline antibiotics. ${ }^{15}$ However, there are also species that we identified that have not been reported to produce antimicrobial agents. Those are for instance, species Streptomyces halotolerance, Streptomyces daliensis and other (as shown in Table 4). This indicates there is strong justification for continuation of our research to discover novel antibiotics from our producer organisms. We proceed with efforts to further characterize these strains and other promising extremophilic actinomycetes in our collection with the ultimate goal to identify the active antibacterial compound from the species that were grown in their specific antibacterial producing growth media.

There are several limitations in this study whereby one of these is the low yield of extracts $(3 \mathrm{~mL}$ of the extract out of 300 $\mathrm{mL}$ of the cultural liquid and hence only $1 \%$ of the total growth media) and hence availability of extracts for testing in our smallscale research. In the future, we will scale up production to enable higher amount of extract production for testing and compound identification. As shown in Table 2, the ZOI when testing our extracts against the MDR pathogens $P$. aeruginosa and $A$. baumannii are not substantial however we are optimistic the results are significant given that we used crude extracts from our producer organisms. We hypothesize that the active compound within our extremophiles actinomycetes producer organism will exhibit potent antibacterial activity once these are identified and isolated into its pure form. In this study, our compound characterization was limited to comparative peak identification of extracts from the same strain grown in different growth media using analytical HPLC and hence no information was made available about the nature and identity of the active compound responsible for the antibacterial activities. However, the analytical HPLC chromatograms generated from this study provided us with an overall profile of the extracts, which have direct correlation with the activity-screening results. The data will guide us in planning for our next steps to achieve our ultimate goal of further characterization and identification of the active antibacterial compounds. Overall, screening of the extracts shows promising results in inhibiting the growth of our test MDR organisms and possibly also other multidrug resistant microorganisms. The extracts that showed the best activity in this study are extracts \# 78, 536, and 550 will be grown in scaled up experiments and further characterized to ensure reproducibility of extract preparation that generate the active material for further study. Chemical characterization for active compound identification will be further enhanced by utilization of state of the art chemistry laboratories available at our Nazarbayev University core facilities that would include use of preparative HPLC, Mass spectrometry and LCMS runs and analyses. At this point in time, our research team has optimized growth culture conditions, extract preparation, activity screening and strain identification protocols for future studies that will guide us in identifying potentially novel compounds from our selected extremophilic strains with activity against important MDR

\section{Conclusions}

Extracts derived from extremophilic actinomycetes have shown significant inhibitory activity against conditionally pathogenic bacteria. Some of the extracts exhibited a broad spectrum of activity inhibiting growth of both gram - positive and gram-negative test bacterial pathogens chosen for our study. On the other hand, there were extracts that only inhibited growth of either gram - positive or gramnegative pathogens. Moreover, there was a difference in activity of the extracts obtained from strains grown in different growth media. HPLC results have shown correlation with the activity of the extracts according to the activity test results. All the chromatograms collectively show a general profile of the extracts and their composition with respect to the growth conditions. Practical analysis shows that growth media affects the production of antibiotic compounds, as either salt or alkalinity can trigger the secretion of the antibiotic by actinomycetes. Identification of a chemical composition of the most potential antibiotic extract would answer most of the questions, such as the nature of the compound, the mechanism of action, and presence or absence of the resistance in pathogenic bacteria. Dereplication process is a target screening method to achieve good quality and quick results in screening and identification of natural antibiotic producer compounds, and will be utilized in our future studies. 


\section{References}

1. American Chemical Society. Discovery and development of penicillin. Available from: http://www.acs.org/content/acs/en/education/whatischemistry/landmarks/flem ingpenicillin.html.

2. Wohlleben W, Mast Y, Stegmann E, Ziemert N. Antibiotic drug discovery. Microb Biotechnol 2016,9:541-8. doi:10.1111/1751-7915.12388.

3. Gebreyohannes G., Moges F, Sahile S, Raja N. Isolation and characterization of potential antibiotic producing actinomycetes from water and sediments of Lake Tana, Ethiopia. Asian Pac J Trop Biomed 2013;3:425-35. doi: 10.1016/S2221-1691(13)60092-1.

4. Basilio A, Gonzalez I, Vicente MF, et al. Patterns of antimicrobial activities from soil actinomycetes isolated under different conditions of $\mathrm{pH}$ and salinity. J Appl Microbiol 2003;95:814-23. doi : 10.1046/j.1365-2672.2003.02049.x.

5. Roberts J, Trenozhnikova L, Seatgon L, et al. Bacterial growth in media mimicking the high salt and alkalinity of extreme Kazakhstan environments results in production of antimicrobial compounds in soil actinomycetes isolated from these extremophile locations. J Pharm Technol Drug Res 2013. doi: http://dx.doi.org/10.7243/2050-120X-
2-14.

6. Santajit S, Indrawattana N. Mechanisms of antimicrobial resistance in ESKAPE pathogens. Biomed Res Int 2016;2016:2475067. doi: $10.1155 / 2016 / 2475067$.

7. O'Neill J. Antimicrobial Resistance: Tackling a crisis for the health and wealth of nations. The review on Antimicrobial Resistance 2014. Avalable from: https://amr-review. org/sites/default/files/AMR\%20Review $\begin{array}{llllllllllll}\% & 2 & 0 & \mathrm{P} & \text { a } & \mathrm{p} & \mathrm{e} & \mathrm{r} & \% & 2 & 0 & -\end{array}$ $\% 20$ Tackling $\% 20 \mathrm{a} \% 20$ crisis $\% 20$ for $\% 2$ 0the $\% 20$ health $\% 20$ and $\% 20$ wealth $\% 20$ of $\% 20$ nations_1.pdf

8. Trenozhnikova. L, Azizan A. Discovery of Actinomycetes from extreme environments with potential to produce novel antibiotics. Cent Asian J Glob Health 2018;7. Available from: file://Users/Francesca/Downloads/3371506-1-PB.pdf

9. Trenozhnikova, L., A. K. Khasenova, A. S. Balgimbayeva, et al. Characterization of the antibiotic compound No. 70 produced by Streptomyces sp. IMV-70. Sci World J 2012 ; $200122: 5944231$. doi:10.1100/2012/594231

10. Andrews JM. BSAC standardized disc susceptibility testing method. J Antimicrob Chemoth 2001;48:43-57. doi: https://doi.org/10.1093/ jac/48. suppl_1.43

11. Abdelfattah MS, Elmallah MYI, Hawas UW, et al.. Isolation and characterization of marine-derived actinomycetes with cytotoxic activity from the Red Sea coast. Asian Pac J Trop Biomed 2016;6: 651-7. Doi: https://doi.org/ 10.1016/j.apjtb.2016.06.004

12. Hamedi J, Mohammadipanah F, Ventosa A. Systematic and biotechnological aspects of halophilic and halotolerant actinomycetes. Extremophiles 2012;17: 1-13. doi: 10.1007/s00792-012-0493-5.

13. Ramya S, Shanmugasundarama $T$, Balagurunathana R. Biomedical potential of actinobacterially synthesized selenium nanoparticles with special reference to anti-biofilm, anti-oxidant, wound healing, cytotoxic and anti-viral activities. J Trace Elem Med Biol 2015;32:30-9.

14. Jiang Y, Cao Y, Wiese J, et al. Streptomyces sparsus sp. nov., isolated from a saline and alkaline soil. Int J Syst Evol Microbiol 2011;61:1601-5.

15. Petković H, Lukežič T, Šušković J. Biosynthesis of oxytetracycline by Streptomyces rimosus: Past, Present and Future Directions in the Development of Tetracycline Antibiotics. Food Technol Biotech 2017;55:3-13. doi:10. 17113/f tb.55.01.17.4617+ 